\title{
Dinámica de la distribución del agua en el sistema de riego Tumbaco en Ecuador
}

\author{
Dynamics of the water distribution in the Tumbaco \\ irrigation system in Ecuador
}

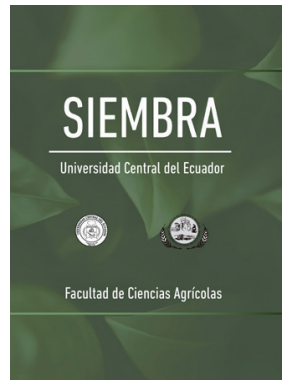

\author{
Blanca Maritza Chile Asimbaya ${ }^{1}$, Randon Stalin Ortiz Calle ${ }^{2}$
}

\footnotetext{
${ }^{1}$ Universidad Central del Ecuador, Facultad de Ciencias Agrícolas, Jerónimo Leiton y Av. La Gasca s/n. Ciudadela Universitaria. 170521 Quito, Pichincha, Ecuador.

凶bmchilea@uce.edu.ec

(D.) https://orcid.org/0000-0002-4496-2653

${ }^{2}$ Universidad Central del Ecuador, Facultad de Ciencias Agrícolas, Jerónimo Leiton y Av. La Gasca s/n. Ciudadela Universitaria. 170521 Quito, Pichincha, Ecuador.

凶rortiz@uce.edu.ec

(D. http://orcid.org/0000-0001-6172-4932
}

*Autor de correspondencia: bmchilea@uce.edu.ec

\section{SIEMBRA}

https://revistadigital.uce.edu.ec/index.php/SIEMBRA

ISSN-e: $2477-8850$

ISSN: $1390-8928$

Periodicidad: semestral

vol. 8, núm. 2, 2021

siembra.fag@uce.edu.ec

DOI: https://doi.org/10.29166/siembra.v8i2.3074

Esta obra está bajo una licencia internacional Creative Commons Atribución-NoComercial

\begin{abstract}
Resumen
La distribución del agua a los usuarios es el resultado de la interacción entre la junta de regantes, los usuarios, la operación y el mantenimiento de la infraestructura hidráulica. El objetivo de la investigación fue identificar los aspectos técnicos y sociales que influyen en la distribución del agua en los ramales principal y secundario del sistema de riego Tumbaco. Se realizó el levantamiento de información de campo a través de encuestas y entrevistas a los actores principales y la medición de caudales en los diferentes ramales. Resultados principales: i) la construcción del sistema de riego empezó en 1909 por los habitantes de Tumbaco; en 1944, la Caja Nacional de Riego concluyó la construcción del canal para cubrir una superficie regable de 2.000 ha; en 2017, la superficie regada fue de 1.050 ha; la junta de usuarios gestiona el sistema desde el año 2001; ii) la infraestructura hidráulica del sistema ha cumplido su vida útil (75 años), no se ha rehabilitado y las compuertas no permiten la regulación de los caudales desde el canal principal a los secundarios; iii) referente a la gestión, los reglamentos no se cumplen. En conclusión, en el sistema de riego Tumbaco no existe equidad en la distribución del agua debido a que la infraestructura del sistema ha cumplido su vida útil, no se cumple con el estatuto ni con el reglamento, y los derechos no se han actualizado con las necesidades de los usuarios, como resultado del proceso existente de desterritorialización.
\end{abstract}

Palabras clave: infraestructura, caudales, junta de regantes, normativa, derechos de agua.

\begin{abstract}
The water distribution to the farmers is the result of the interaction between the irrigation water association, farmers, operation and maintenance of the hydraulic infrastructure. The objective of the research was to identify technical and social aspects linked to the water distribution at the secondary branch canals of the Tumbaco irrigation system. Field information was collected through surveys and interviews to the main actors and measurement of flows in the canals. The main results: i) the construction of the irrigation system began in 1909 by the inhabitants of Tumbaco; in 1944, the "Caja Nacional de Riego" completed the construction of the canal to cover an irrigable area of 2,000 ha; in 2017, the irrigated area was 1,050 ha; the water user board has managed the system since 2001; ii) the hydraulic infrastructure of the system has reached its time life ( 75 years), it has not been rehabilitated and the gates does not allow the regulation of the discharges from the main to the secondary canals; iii) regarding the administration, the regulations are not followed in practice. In conclusion, in the Tumbaco irrigation system there is no equity in the water distribution to the farmers because the infrastructure of the system has reached its lifetime, the statute and regulation norms are not accomplished, the rights have not been updated to the needs of the users as a result of the existing process of deterritorialization.
\end{abstract}

Keywords: infrastructure, flows, board of irrigators, regulations, water rights. 


\section{Introducción}

La variación del clima - especialmente la temperatura - ha mantenido una tendencia creciente en los últimos 60 años, y según los escenarios del cambio climático esta tendencia se mantendrá (Chesini, 2018). En Quito, las temperaturas medias se han incrementado en $1,2^{\circ} \mathrm{C}$, las mínimas en $1,1^{\circ} \mathrm{C}$ y las máximas en $0,7^{\circ} \mathrm{C}$, paralelamente con el incremento de la temperatura, la evapotranspiración también se ha incrementado (Sarricolea et al., 2017). La precipitación ha disminuido en $7 \mathrm{~mm}$ por mes (Hernández, 2016). El incremento de la evapotranspiración y la reducción de la precipitación afectarán a la disponibilidad del agua para el consumo humano, agrícola e industrial, dando como resultado una mayor presión sobre los recursos hídricos (Chávez-Jiménez y González-Zeas, 2015). En consecuencia, la realidad actual demanda de un uso optimizado del agua de riego considerando que el sector agrícola utiliza alrededor del $80 \%$ del agua dulce.

El riego es fundamental para la seguridad alimentaria. Los sistemas de riego han aportado a la producción agropecuaria, transformado el paisaje y mejorado la productividad (Gallardo, 1987).

Uno de los parámetros más importantes del funcionamiento de un sistema de riego es la distribución del agua, la cual depende del manejo y operación de la infraestructura hidráulica, de las actividades sociales de los usuarios y de los mecanismos, criterios y reglas que rigen la distribución de agua, es decir, cómo se establecen los derechos de usos de agua, turnos de riego, caudales de entrega, entre otros (Gutiérrez y Gerbrandy, 1998).

En la infraestructura de riego existen elementos importantes a considerar como: áreas de riego, red de canales y de tuberías, caudal de operación, sistemas de distribución del agua, estructuras hidráulicas tanto en canales como en tuberías y métodos de aplicación del agua (Ortiz, 2019). La función de la organización de regantes es indispensable para garantizar la operatividad de un sistema de riego y de las modalidades colectivas de gestión y administración del agua (Apollin y Eberhart, 1998). La organización de regantes es un elemento fundamental del sistema de riego debido a que define la normatividad, estatuto y reglamento para la distribución interna del agua (Tamayo, 2015).

La normatividad es el conjunto de reglas y procedimientos que rigen la distribución del agua de riego, es decir, el acuerdo sobre cómo se establecen los derechos colectivos e individuales para el acceso al agua, estructura de la organización para la distribución del agua y participación de los miembros en el control (Olazaval et al., 1999).

En Ecuador, entre los años 1990 y 2000, los sistemas de riego públicos fueron transferidos a las juntas de regantes: de 76 sistemas de riego públicos, 40 fueron transferidos a las juntas de usuarios para su administración, operación y mantenimiento (Valdivieso Armijos, 2013). En la provincia de Pichincha, el área bajo infraestructura de riego es de 81.365 ha, el $16 \%$ se encuentra con infraestructura construida por el Estado, la cual corresponde a los sistemas públicos transferidos a los usuarios (Gobierno Autónomo Descentralizado de la Provincia de Pichincha [GADDP], 2013).

El sistema de riego Tumbaco es un sistema de riego público transferido. La gestión del sistema estuvo a cargo de la Caja Nacional de Riego (entre 1947 y 1966), posteriormente por el ex-Instituto Ecuatoriano de Recursos Hidráulicos (INERHI) entre 1966 y 1990. En el marco de las políticas de ajuste estructural de los años 1990, el INERHI fue reemplazado por el Consejo Nacional de Recursos Hídricos (CNRH), creándose las Corporaciones Regionales de Desarrollo en lo relacionado al estudio, construcción y gestión de los sistemas de riego públicos. De esta manera, el sistema de riego Tumbaco pasó a ser administrado por la Corporación de Desarrollo Regional Sierra Norte (CORSINOR) para luego ser transferido a la Junta General de Usuarios en el marco del Proyecto de Asistencia Técnica en Riego "UEP-PAT". A partir del año 2001, la Junta General de Usuarios ha sido la encargada de la gestión del sistema (Tamayo, 2016).

El sistema de riego Tumbaco se encuentra afectado por la acelerada urbanización de las tierras agrícolas, esto ha influenciado en su gestión, principalmente en la distribución del agua en los distintos ramales y unidades terciarias que conforman el sistema. Desde 1994 hasta 2017, el área agrícola ha disminuido en un 50 \%; el número de usuarios también ha disminuido en un $25 \%$ (algunos usuarios vendieron sus propiedades, mientras que otros han fraccionado sus tierras para entregarlas como herencia a sus hijos), generando inconvenientes en la operación, gestión y distribución de agua, así como los siguientes problemas:

- El caudal de autorización de uso y aprovechamiento fue entregado en un inicio para cubrir una superficie de 2.115 ha. En la actualidad, se sigue derivando de los ríos Pita y Guangal un caudal de 1,64 $\mathrm{m}^{3} \mathrm{~s}^{-1}$ para irrigar 1.050 ha, ocasionando desbordamientos por efecto de la reducción de la sección y cambio en el trazado de los canales para dar paso a las urbanizaciones, sistemas de alcantarillado y pavimentación de vías. 
- En la red de riego, la infraestructura existente no permite regular el caudal a entregarse a los diferentes ramales en función de la necesidad real de agua, lo cual afecta a la distribución del agua.

- Los agricultores se encuentran afectados por el mantenimiento pobre del sistema de riego, baja eficiencia de distribución del agua, conflictos entre usuarios, robo del agua, no se respetan los turnos de riego, entre otros problemas de orden social, los cuales inciden en la distribución y uso eficiente del agua.

La Facultad de Ciencias Agrícolas de la Universidad Central del Ecuador, a través del Centro Académico Docente Experimental La Tola (CADET), es uno de los principales usuarios del sistema de riego Tumbaco, con derechos y obligaciones (pago de tarifas, asistencia a mingas, entre otros) para la gestión del riego y como entidad académica con visión socio-técnica, debe tener un vínculo directo en la generación de conocimiento que conduzca a solventar la problemática que afecta al territorio de influencia del sistema de riego Tumbaco (Tamayo, 2016). A través del área de vinculación, en el año 2015 se firmó el "Convenio entre la Facultad de Ciencias Agrícolas de la Universidad Central del Ecuador y la Junta General de Usuarios del Sistema de Riego Tumbaco", como un primer resultado se obtuvo el catastro actualizado del sistema. En una segunda fase, con el proyecto "Fortalecimiento de la gestión del sistema de riego Tumbaco", se realizó la elaboración de turnos de riego, diseño de estructuras hidráulicas, estudios de calidad de agua, mejoramiento de las capacidades para el aprovechamiento del agua y evaluación de la eficiencia del sistema de riego.

Con estos antecedentes, el objetivo de la investigación fue analizar la dinámica de la distribución del agua en el sistema de riego Tumbaco, integrando los aspectos técnicos y sociales, para determinar elementos que permitan mejorar la gestión del riego.

\section{Materiales y métodos}

\subsection{Materiales}

La zona de estudio se encuentra ubicada en la parte alta de la cuenca del río Esmeraldas, en la microcuenca del río Pita, cantón Quito, parroquias Tumbaco, Alangasí y La Merced, entre las cotas 2.300 m s. n. m. y 2.700 m s. n. m. (Figura 1).

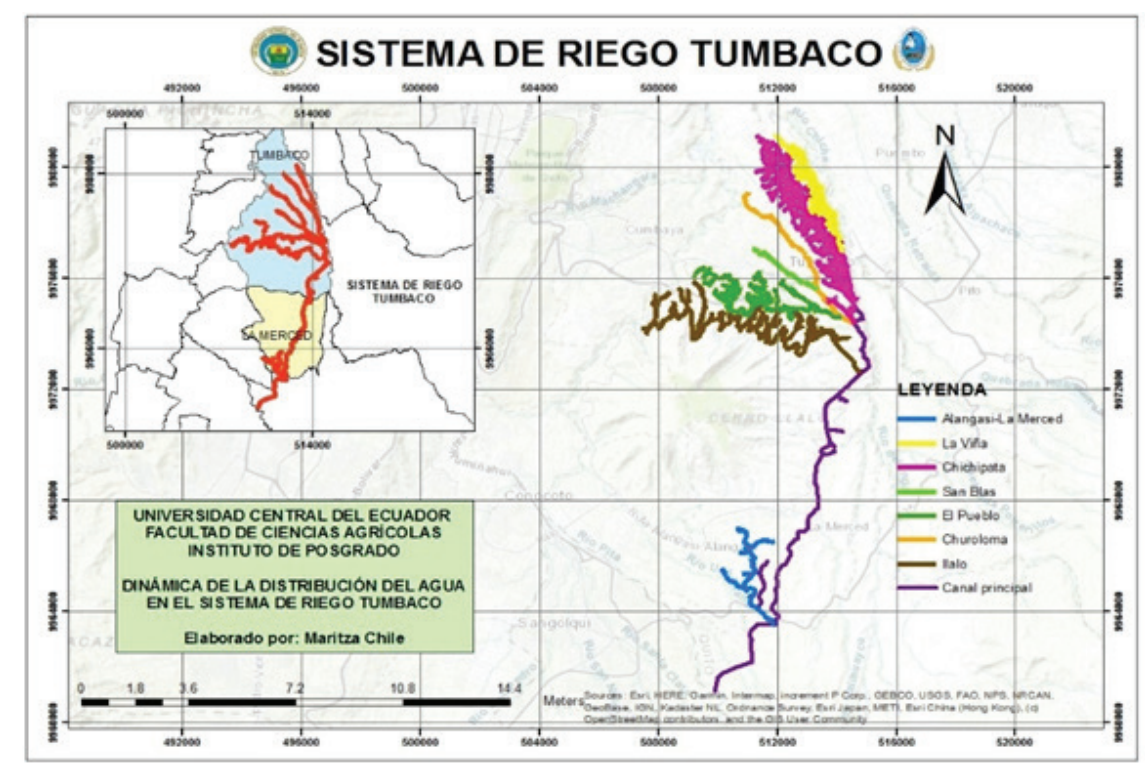

Figura 1. Ubicación geográfica del sistema de riego Tumbaco.

Figure 1. Geographic location of the Tumbaco irrigation system.

Las condiciones agroecológicas del valle de Tumbaco se caracterizan por tener dos estaciones climáticas bien diferenciadas: la lluviosa de octubre a mayo, y verano de junio a septiembre (Figura 2) (Ortiz y Chile, 2020). La precipitación promedio anual de la serie histórica de 1986 hasta el 2018 es de 852 mm, mientras que la eva- 
potranspiración promedio anual es de $1.152 \mathrm{~mm}$ (Instituto Nacional de Metereología e Hidrología [INAMHI], 2018). Durante el mes de agosto existe la mayor evapotranspiración mensual con 136,65 mm y la máxima necesidad hídrica diaria con un valor máximo de 4,28 mm (Ortiz y Chile, 2020).

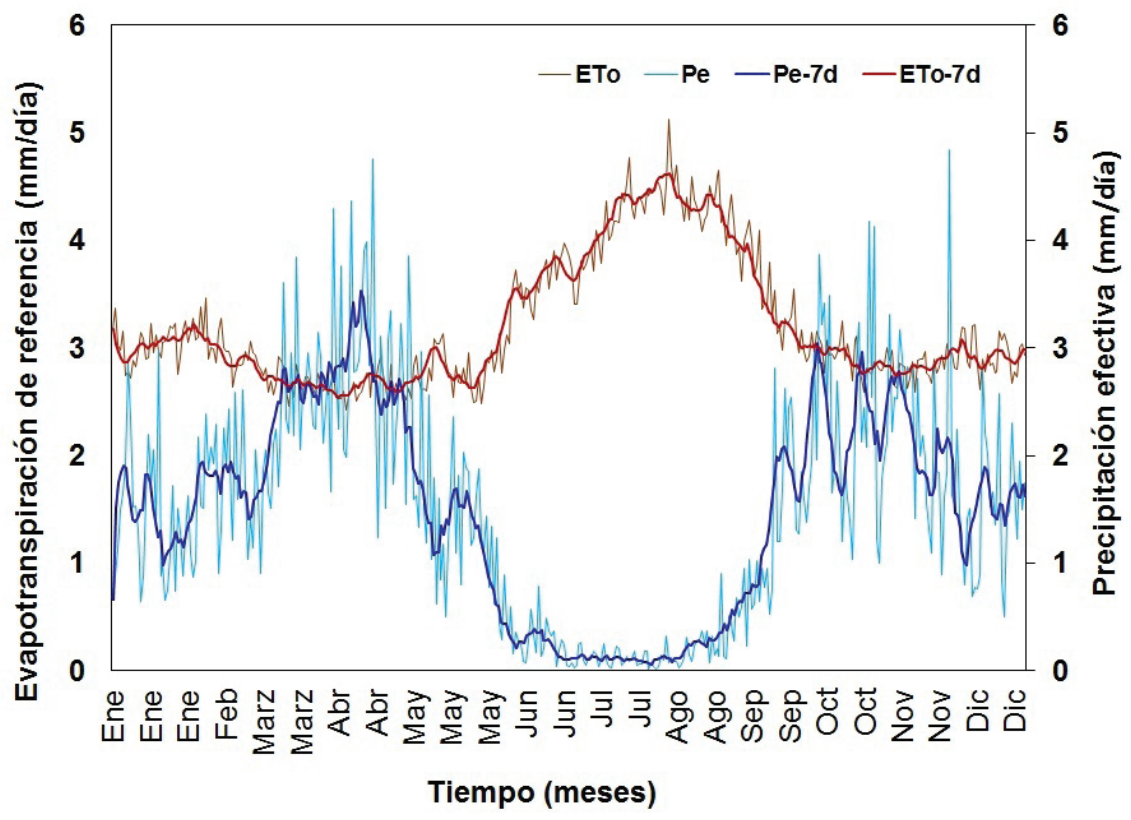

Figura 2. Evapotranspiración y precipitación para la estación La Tola, años 1986-2018 (INAMHI, 2018).

Figure 2. Evapotranspiration and precipitaction for La Tola station, years 1986-2018 (INAMHI, 2018).

\subsection{Métodos}

En la investigación se realizó un estudio de caso, a través de metodologías cuantitativas y cualitativas (Martínez, 2011). Las etapas del estudio fueron: selección de la unidad de estudio, planteamiento del problema, revisión de literatura, elaboración de objetivos, variables, instrumentos, recopilación de datos, codificación y análisis de la información (Tamayo, 2004; Martínez Carazo, 2006).

La información referente al sistema de riego Tumbaco fue proporcionada por el directorio de la Junta de Regantes, como: redes de canales, planos, mapas, estatuto, reglamentos, turnos de riego, padrón de usuarios, entre otra información. Se utilizó el siguiente procedimiento para el desarrollo de la investigación:

1. Para el análisis de la historia de riego se realizó la revisión de archivos históricos, padrón de usuarios de la junta de riego, estatutos y reglamentos de la junta, autorizaciones de uso y aprovechamiento y entrevistas a las personas adultas en cada ramal del sistema de riego Tumbaco.

2. Se realizaron recorridos por la red de canales secundarios para verificar el catastro realizado en 2017 para la caracterización física de la red de riego.

3. Se utilizó el medidor Parshall (método preciso) para la medición de caudales en los ramales, así como el caudal de entrega en función de las necesidades hídricas de los cultivos y del área de riego. Con base en estos parámetros se determinó la eficiencia de conducción y de distribución, así como la equidad en la distribución del agua. La medición de los caudales se realizó a la entrada de cada ramal para contrastar el caudal medido con el planificado en octubre 2018, julio 2019 y septiembre 2019. En el medidor Parshall se realizó la medición de la carga hidráulica aguas arriba de la garganta, con un total de cinco lecturas consecutivas con intervalos de tiempo de cinco minutos entre lecturas.

El método Parshall es un método técnicamente descrito como un canal Venturi, para medir el caudal en cauces abiertos. El funcionamiento de este aforador se basa en generar un flujo crítico estrechando la anchura de la garganta de la canaleta y levantando la base; este efecto obliga al agua a elevarse, proceso que debido a la aceleración del flujo permite establecer una relación matemática entre la altura del agua y el gasto (Tovar y Anyela, 2020).

4. Para el análisis de las normas, derechos y relaciones sociales relacionados con la distribución del agua, se realizaron entrevistas al directorio de la junta, canaleros, presidentes de los ramales, presidentes de los 
óvalos y encuestas a los usuarios, con una muestra de 56 representantes de los presidentes de los óvalos y 93 usuarios del sistema de riego, representando el $5 \%$ de la población total (usuarios).

\section{Resultados}

Los moradores de Tumbaco empezaron la construcción de la red de canales en 1909. El trabajo inició en la hacienda del colegio Alangasí, construyéndose cinco kilómetros. En 1918 reanudaron el trabajo y con grandes sacrificios lograron construir tres $\mathrm{km}$ adicionales. Los usuarios abandonaron la construcción del canal alrededor de 12 años, y en 1930 acudieron al Congreso Nacional para, mediante impuestos, culminar la construcción del canal. El impuesto inicial creado fue de seis sucres por hectárea y luego de dos años, un impuesto complementario de dos sucres por hectárea, con estos fondos se realizó la construcción del sistema (Herreras, 1991). En 1944, se creó la Caja Nacional de Riego, institución que en el período 1944-1947 concluyó con la construcción del canal planificado inicialmente, en donde se incluyó la captación del río Pita (Pons, 1948; Carrera, 2015). En 1973, el sistema cubrió una extensión de 2.300 ha, con un canal principal de 20,6 km y de 35,5 km de canales secundarios, en los siguientes ramales: Churoloma con 22 óvalos, Chichipata con 30 óvalos, San Blas con 14 óvalos y El Pueblo con 33 óvalos (Instituto Ecuatoriano de Recursos Hidraúlicos [INERHI], 1973). En el año 1994 se tuvo 2.115 ha regadas en los siete ramales, según los archivos de la Junta General de Usuarios. Desde ese año se cuenta con siete ramales en el sistema.

En 1948, el área regable inicial fue estimada en 2.000 ha; en 1973, se incrementó a 2.300 ha; en 1994, se redujo a 2.115 ha; en 2008 a 1.910 ha y en el 2017 a 1.050 ha (Pons, 1948; INERHI, 1973; Montúfar, 2017; Junta de Riego Tumbaco [JGUSIRTUM], 2019). En 1973 el sistema benefició a 1.100 familias y en el 2017 a alrededor de 2.499 usuarios, como producto de la división de las tierras por herencias.

En 1940, los cultivos con mayor superficie fueron: maíz, arveja, vainita, alfalfa, lechugas, entre otras legumbres. En 1960 se convierte el área agrícola en grandes huertos frutales, predominando la producción de aguacate. Entre 1960 y 1970, muchos huertos se convirtieron en quintas. En 1970, se inicia la siembra de ornamentales como buganvilla, cucardas y rosas (Sánchez, A., comunicación personal, 17 de agosto de 2019).

El canal principal tiene una longitud de 21,76 km. Los ramales secundarios suman 48,61 km; ramal Alangasí La Merced, 3,83 km; ramal Ilaló, 13,65 km; ramal Chichipata, 8,63; ramal Churoloma, 6,95 km; ramal el Pueblo, 8,16 km; ramal La Viña 4,36 km y ramal San Blas 2,99 km. La infraestructura hidráulica cuenta con 75 años de uso, se encuentra totalmente deteriorada y las compuertas no permiten regular caudales desde el ramal principal a los ramales secundarios. La mayoría de los usuarios califican el estado de la infraestructura como regular.

En 1947, la concesión del agua de riego fue de $1.2001 \mathrm{~s}^{-1}$ (dotación de $0,771 \mathrm{~s}^{-1} \mathrm{ha}^{-1}$ ) y en 1994, de 1.640 $1 \mathrm{~s}^{-1}$, la cual se ha mantenido hasta la actualidad (Tabla 1).

Tabla 1. Superficie regada y caudal teórico en los ramales

Table 1. Irrigated area and theoretical flow in the branches

\begin{tabular}{lccccc}
\hline \multicolumn{1}{c}{ Ramal } & SR 1994 & QR 1994 & SR 2017 & QR 2019 & QC \\
\hline Principal & 87,0 & 67,4 & 94,0 & - & \\
Alangasí & 138,5 & 107,3 & 10,8 & 100,0 & \\
Ilaló & 334,0 & 258,8 & 231,7 & 320,0 & \\
Chichipata & 519,5 & 402,6 & 300,4 & 350,0 & $1.640,0$ \\
La Viña & 170,0 & 131,7 & 88,3 & 150,0 & \\
El Pueblo & 405,0 & 313,8 & 145,0 & 150,0 & \\
Churoloma & 315,0 & 244,1 & 153,9 & 180,0 & \\
San Blas & 146,75 & 113,7 & 31,1 & 120,0 & \\
\hline Total & $2.115,75$ & $1.639,7$ & $1.055,6$ & $1.370,0$ & \\
\hline
\end{tabular}

SR: superficie de riego (ha); QR: caudal de riego $\left(1 \mathrm{~s}^{-1}\right)$; QC: caudal concesionado $\left(1 \mathrm{~s}^{-1}\right)$ 
Según los caudales medidos, el caudal para todo el proyecto en octubre de 2018 fue de 1.443,3 $1 \mathrm{~s}^{-1}$, en julio de 2019 de $1.244,51 \mathrm{~s}^{-1}$ y en septiembre de 2019 de 1.098,7 $1 \mathrm{~s}^{-1}$ (Tabla 2 y Figura 3). Los caudales entregados por la Junta a los ramales son los mismos durante los 365 días del año, sin embargo, la precipitación, la evapotranspiración y las necesidades hídricas de los cultivos varían mensualmente. En el ramal Alangasí-La Merced, el caudal máximo requerido es de $11,211 \mathrm{~s}^{-1}$, sin embargo, el caudal promedio medido fue de 64,82 $1 \mathrm{~s}^{-1}$, existiendo un exceso de cinco veces más de lo requerido, mientras que la Junta teóricamente asigna un caudal de $1001 \mathrm{~s}^{-1}$ (Tabla 2).

Tabla 2. Caudales medidos durante la fase de campo $\left(1 \mathrm{~s}^{-1}\right)$

Table 2. Flow rates measured during the field phase $\left(l \mathrm{~s}^{-1}\right)$

\begin{tabular}{lcccccc}
\hline \multicolumn{1}{c}{ Ramal } & QR 2017 & Q1 & Q2 & Q3 & Qm & QA \\
\hline Alangasí & 11,21 & 74,27 & 63,5 & 56,7 & 64,82 & 100,0 \\
Ilaló & 271,37 & 264,0 & 220,5 & 220,5 & 235,00 & 320,0 \\
Chichipata & 315,67 & 237,0 & 234,0 & 210,0 & 227,00 & 350,0 \\
El Pueblo & 151,00 & 254,0 & 263,0 & 175,0 & 230,67 & 150,0 \\
Churoloma & 160,09 & 257,0 & 210,0 & 183,0 & 216,67 & 150,0 \\
San Blas & 29,10 & 49,16 & 40,2 & 55,0 & 48,12 & 180,0 \\
La Viña & 93,11 & 250,0 & 222,5 & 201,6 & 224,70 & 120,0 \\
\hline Total & 749,03 & $1.443,3$ & $1.244,5$ & $1.098,7$ & & $1.370,0$ \\
\hline
\end{tabular}

QR: caudal requerido; Q1: caudal aforado en octubre 2018; Q2: caudal aforado en julio 2019; Q3: caudal aforado en septiembre 2019; Qm: caudal medio; QA: caudal asignado por la junta.

En el ramal Ilaló, el caudal máximo requerido en el mes de julio es de 213,98 $1 \mathrm{~s}^{-1}$, el caudal entregado por la Junta de $350,01 \mathrm{~s}^{-1}$ y el caudal medido de 220,0 1 s'-1, existiendo un exceso del 2,8\%. En el ramal Chichipata, el caudal máximo de demanda en el mes de julio fue de 237,38 $1 \mathrm{~s}^{-1}$, el caudal entregado por la Junta de 320,0 $1 \mathrm{~s}^{-1} \mathrm{y}$ el caudal medido de 234,0 $1 \mathrm{~s}^{-1}$, existiendo un déficit del 1,4\%. En el ramal El Pueblo, el caudal máximo requerido fue de $151,01 \mathrm{~s}^{-1}$, el caudal entregado por la Junta de $150,01 \mathrm{~s}^{-1} \mathrm{y}$ el caudal promedio medido de $230,671 \mathrm{~s}^{-1}$, con un exceso del $52 \%$. En el ramal Churoloma, el caudal máximo requerido fue de 160,09 $1 \mathrm{~s}^{-1}$, el caudal entregado por la Junta de 150,0 $1 \mathrm{~s}^{-1}$, y el caudal promedio medido de $216,671 \mathrm{~s}^{-1}$, existiendo un exceso del 35,34 \%. En el ramal San Blas, el caudal requerido fue de $29,11 \mathrm{~s}^{-1}$, el caudal entregado por la Junta de $180,01 \mathrm{~s}^{-1}$, el caudal promedio medido fue de 48,12 $1 \mathrm{~s}^{-1}$, con un exceso del 65,30\%. En el ramal La Viña, el caudal requerido fue de $93,111 \mathrm{~s}^{-1}$, el caudal entregado por la Junta de $180,01 \mathrm{~s} \mathrm{~s}^{-1}$, el caudal promedio medido de $224,701 \mathrm{~s}^{-1}$, con un exceso del $141,0 \%$ (Tabla 2 ).

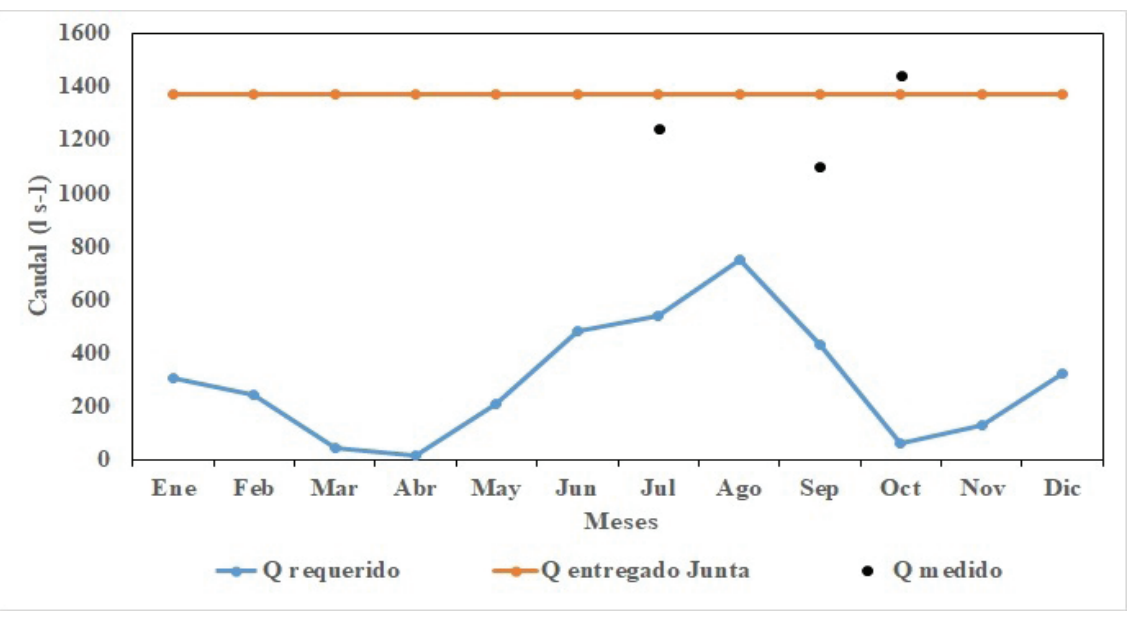

Figura 3. Caudales en el sistema de riego Tumbaco.

Figure 3. Discharges in the Tumbaco irrigation system. 
La Junta de Riego es la organización que administra, opera y mantiene el sistema de riego, está constituida por: Asamblea General de Usuarios, Directorio Central, directivas de ramales y óvalos, y la Comisión Electoral. Según el estatuto y reglamento de operación y mantenimiento, uno de los objetivos principales es vigilar la correcta distribución del agua y el cumplimiento de todas las normas, para asegurar una distribución equitativa del agua a los usuarios (JGUSIRTUM, 2017). La tarifa que pagan los usuarios sirve para el pago del personal administrativo, canaleros, obras y para cancelar el uso de aprovechamiento del agua. Para operar el sistema de riego, la Junta cuenta con 4 canaleros, encargados de realizar los recorridos por los siete ramales, de lunes a domingo, de 7:30 a. m. a 4:00 p. m., para asegurar que no existan problemas en el funcionamiento de la red y garantizar la entrega del agua a los usuarios. Se realiza el mantenimiento preventivo desde la captación hasta la Facultad de Ciencias Agrícolas (canal principal) a través de una tarifa especial, mientras que para el mantenimiento de los ramales se realizan mingas, con la finalidad de mantener las relaciones sociales y conservar las costumbres ancestrales.

El 48,5\% de los usuarios entrevistados indican que el trabajo realizado por el presidente de la Junta es regular, el 30,1 \% bueno y el 15,5\% malo. En relación con los presidentes de los ramales, 33,54 \% es bueno, $26,5 \%$ regular, $17,8 \%$ malo y el $22,1 \%$, no conocen el rol que realiza el presidente del ramal.

Respecto a las reglas de distribución del agua, la Junta establece un tiempo de entrega del agua o turno de riego en 12 horas por hectárea y es proporcional a la superficie del usuario. La frecuencia de entrega del agua es de siete días. Para mantener el derecho al turno de riego, según el reglamento y el estatuto, la Junta ha planteado tarifas y mingas. Respecto a las actividades que realizan para mantener el derecho, el $87 \%$ realizan mingas, $6 \%$ pagan una tarifa anual, $5 \%$ en el mantenimiento de sus óvalos y el $2 \%$ en otras actividades.

\section{Discusión}

La construcción del sistema de riego en este territorio empezó con la voluntad de un pueblo, que unió esfuerzos (económicos, humanos y materiales) para construir un canal que permita transportar el agua de riego hasta sus parcelas para mejorar la producción de sus cultivos. Sin embargo, pese a su esfuerzo, se necesitaba de fondos extras para concluir con la construcción del canal, viéndose obligados a acudir al Congreso Nacional para que a través de una ley se asignen recursos económicos por la construcción del canal, creándose la Caja Nacional de Riego para tal efecto. Este canal de riego se construyó técnicamente en 1944, aun cuando no existían estaciones climatológicas en el entorno para determinar las necesidades hídricas de los cultivos, no se conocía mucho en el medio sobre las estructuras hidráulicas óptimas para regular los caudales entre ramales y tampoco se conocía sobre los caudales que debían asignarse técnicamente a los agricultores para operar el riego por surcos. Aspectos técnicos que hoy en día afectan a la distribución de caudales entre ramales y desde los canales secundarios a las unidades terciarias.

Durante los trabajos de urbanización se afectó a la red de canales del sistema de riego debido a la canalización y entubados de ciertos tramos sin diseño hidráulico. Tanto el Municipio como los usuarios y las constructoras, por desconocimiento de la ley, no cumplieron con el art. 62 del Reglamento a la Ley de Recursos Hídricos Usos y Aprovechamiento del Agua en el territorio, el cual estipula que a cinco metros de los márgenes derecho e izquierdo del canal no se puede realizar ninguna construcción: "Los terrenos que lindan con los cauces están sujetos en toda su extensión longitudinal a una zona de servidumbre para uso público. La extensión de la zona de servidumbre será de cinco metros pudiéndose variar, en más o en menos, por razones topográficas, hidrográficas o por las necesidades concretas del otorgamiento de autorizaciones de uso de agua o de aprovechamiento productivo de agua".

El área de riego se redujo de 2.300 ha a 1.050 ha, sin embargo, el caudal asignado sigue siendo el mismo, exceso de agua que ocasiona inundaciones en la parte baja por el desbordamiento que ocurre en los canales de riego. Para regular la asignación, la Secretaría Nacional del Agua debería aplicar el art. 14. de la Ley Orgánica de Recursos Hídricos.

La distribución del agua en el sistema de riego no es equitativa, afectada principalmente por la tipología de las estructuras hidráulicas, las cuales no permiten regular los caudales a lo largo del año. Esta falta de regulación de caudales afecta a los usuarios con sobre y subirrigación, ocasionando graves afectaciones a los usuarios que no reciben la cantidad adecuada de agua para la producción de cultivos, creando conflictos entre usuarios y con la dirigencia de la junta de regantes, todo como resultado de la falta de un departamento de ingeniería dentro de la junta, que permita planificar la distribución del agua en forma permanente. El Estado, como planificador y regulador del reparto de agua, debe garantizar una distribución justa del agua. 
La normativa nacional garantiza las competencias de las juntas de riego, sin embargo, la junta de riego Tumbaco debe mejorar la ejecución de sus competencias, encontrar mecanismos para socializar el trabajo realizado, pues no todos los usuarios conocen el mismo. Una de las debilidades es que existe poco interés de los socios por pertenecer a una directiva tanto de los óvalos como de los ramales, debido a que tienen otras actividades que les generan mejores beneficios económicos que la agricultura. En cuanto a los derechos individuales, la falta de conocimiento técnico ha influido en una mala operación de los turnos de riego, pues los derechos no son proporcionales según los regímenes temporales y la cantidad varía entre lo oferta y la demanda de agua, causando una distribución pobre del agua.

\section{Conclusiones}

El sistema de riego Tumbaco tiene varios hitos históricos, que empezaron desde su construcción, el cual tuvo un aporte significativo del pueblo tumbaqueño. Con el apoyo de la Caja Nacional de Riego se logró concluir la obra en el año 1944; la disminución de la superficie agrícola se produjo por la urbanización de los terrenos agrícolas, siendo éste un proceso de desterritorialización para los campesinos y reterritorialización con las relaciones de poder de los nuevos actores de la ciudad existentes en el territorio de influencia del sistema.

La infraestructura del sistema se ha deteriorado por los años transcurridos desde su construcción (75 años) y no permite la regulación de caudales, lo que influye en la inequidad de la distribución del agua del canal principal a los ramales secundarios y terciarios. La distribución de caudales hacia los ramales secundarios es inequitativa, en seis de los siete ramales existe un exceso, mientras que en el ramal con mayor vocación agrícola se presenta un déficit en la época de verano, existe una brecha entre lo requerido, lo planificado y los caudales reales medidos, mientras que, a nivel de óvalos, los turnos son entregados de acuerdo a la superficie, pero no al requerimiento real del usuario.

El rol de la organización social se vuelve fundamental para la sostenibilidad del sistema de riego, a través de la participación de actores como presidentes de la junta de los ramales, de los óvalos y canaleros; sin embargo, el rol de estos actores no es calificado como bueno por todos los usuarios, y esto repercute en la sostenibilidad de la junta, por la falta del aporte colectivo. La conservación y actualización de los derechos es clave para disminuir la inequidad en la distribución de agua; además, estos derechos del agua deben estar claramente definidos en la Ley Orgánica de Recursos Hídricos Usos y Aprovechamiento del Agua.

A través de este estudio de caso se muestra cómo la noción de equidad varía dependiendo de los actores. Por un lado, el presidente de la junta trata de ser equitativo de acuerdo con la superficie del terreno, sin tomar en cuenta cuánta agua realmente llega al usuario, mientras que para algunos usuarios la distribución no es justa. El rol de actores externos también es importante y es aquí donde se observa la falta de una política clara para el riego en el Ecuador; varias de las instituciones creadas no cumplen su rol en la práctica, por ejemplo, SENAGUA [Secretaría del Agua] con la entrega de sobre concesiones, el GAD [Gobierno Autónomo Descentralizado] no cumple con todas las funciones para el apoyo en la cogestión y el MAG [Ministerio de Agricultura] con un aporte pobre en la tecnificación del riego parcelario.

Se determinó que la infraestructura, la medición periódica de caudales, el rol de la junta y la participación de los usuarios son factores fundamentales para la sostenibilidad del sistema de riego.

\section{Contribuciones de los autores}

- Blanca Maritza Chile Asimbaya: conceptualización, análisis formal, investigación, metodología, administración del proyecto, recursos, visualización, redacción borrador original.

- Randon Stalin Ortiz Calle: conceptualización, metodología, administración del proyecto, supervisión, redacción - revisión y edición.

\section{Referencias}

Apollin, F., \& Eberhart, Ch. (1998). Metodologías de análisis y diagnóstico de sistemas de riego campesino. CAMAREN. CESA. CICDA - RURALTER. 
Carrera, G. (2015). Tumbaco de antaño. Da Vinci Corporación Gráfica.

Chávez-Jiménez, A., \& González-Zeas, D. (2015). El impacto de los caudales medioambientales en la satisfacción de la demanda de agua bajo escenarios de cambio climático. Ribagua, 2(1), 3-13. https:// doi.org/10.1016/j.riba.2015.04.001

Chesini, F. (2018). Los desafíos sanitarios del cambio climático en Argentina. Ingeniería sanitaria y ambiental, 134, 34-37.

Gallardo, G. (1987). Políticas de riego en el Ecuador. En E. Kingman (ed.), Riego en los Andes ecuatorianos (pp. 87-98). CAAP. http://hdl.handle.net/10469/9892

Gobierno Autónomo Descentralizado de la Provincia de Pichincha [GADDP]. (2013). Diagnóstico de riego y drenaje de la provincia de Pichincha. GADDP. http://sitp.pichincha.gob.ec/repositorio/diseno_paginas/ archivos/Diagn\%C3\%B3stico\%20de\%20Riego\%20y\%20Drenaje\%20de\%201a\%20provincia\%20 de\%20Pichincha.pdf

Gutiérrez, Z., \& Gerbrandy, G. (1998). Distribución del agua, organización social y equidad en el pensamiento andino. En R. Boelens \& G. Dávila (eds.), Buscando la equidad: concepciones sobre justicia y equidad en el riego campesino (pp. 259-268). Van Gorcum.

Hernández, Y. (2016). Efectos del cambio climático en registros de temperatura y precipitación en la ciudad de Quito, Ecuador. Escuela Politécnica Nacional.

Herreras, J. E. (1991). Monografía de la parroquia de Tumbaco. Talleres Abya Yala.

Instituto Ecuatoriano de Recursos Hidraúlicos [INERHI]. (1973). Proyectos del INERHI. Biblioteca Ecuatoriana Aurelio Espinosa Pólit.

Instituto Nacional de Meteorología e Hidrología [INAMHI]. (2018). Datos climáticos. INAMHI.

Junta de Riego Tumbaco [JGUSIRTUM]. (2017) Estatuto y Reglamento de Operación y Mantenimiento de la Junta de Riego Tumbaco, 2017. JGUSIRTUM.

Junta de Riego Tumbaco [JGUSIRTUM]. (2019) Archivos de la Junta, 2019. JGUSIRTUM.

Martínez Carazo, P. C. (2006). El método de estudio de caso: estrategia metodológica de la investigación científica. Pensamiento y Gestión, 20, 165-193. https://rcientificas.uninorte.edu.co/index.php/ pensamiento/article/view/3576

Martínez, J. (2011). Métodos de investigación cualitativa. Silogismo 1(8), 25-38. http://www.cide.edu.co/ojs/ index.php/silogismo/article/view/64/53

Montúfar, C. (2017). Catastro del sistema de riego Tumbaco. Universidad Central del Ecuador.

Olazaval, H., Noordholland de Jong, J., \& Alvear, J. (1999). Infraestructura del riego. Elementos técnicos y sociales. INDUGRAF del Ecuador.

Ortiz, R. (2019). Guía Metodologica. Infraestructura de riego de la Escuela Nacional de Irrigación Productiva. Ministerio de Agricultura, Acuacultura y Pesca.

Ortiz, R. S., \& Chile A. M. (2020). Métodos de cálculo para estimar la evapotranspiración de referencia para el Valle de Tumbaco. Siembra, 7(1), 070-079. https://doi.org/10.29166/siembra.v7i1.1450

Pons, J. (1948). Fomento de la Agricultura y la Caja Nacional de Riego. Biblioteca Ecuatoriana Aurelio Espinosa Pólit.

Sarricolea, P., Ruiz, Ó. M., \& Aravena, H. (2017). Tendencias de la precipitación en el norte grande de Chile y su relación con las proyecciones de cambio climático. Diálogo Andino, 54, 41-50. http://dx.doi. org/10.4067/S0719-26812017000300041

Tamayo, C. (2015). Análisis de las economías campesinas en la acequia Mocha-Huachi. Provincia de Tungurahua. Universidad Central del Ecuador.

Tamayo, C. (2016). Caracterización básica del área de influencia del sistema de riego Tumbaco. Universidad Central del Ecuador

Tamayo, M. (2004). El proceso de la investigación cientifica. Limusa / Noriega Editores.

Tovar, L., \& Anyela, R. (2020). Calibración de la canaleta Parshal. Universidad Cooperativa de Colombia.

Valdivieso Armijos, S. (2013). Análisis de un proceso de desconcentración y descentralización en el Ecuador: estudio de caso. "La transferencia de la competencia de riego en la provincia de Pichincha. IAEN Instituto de Altos Estudios Nacionales - Universidad de Posgrado del Estado. 